\title{
Erratum: Half-supersymmetric solutions in five-dimensional supergravity
}

\author{
Jan B. Gutowski ${ }^{a}$ and Wafic Sabra ${ }^{b}$ \\ ${ }^{a}$ DAMTP, Centre for Mathematical Sciences, University of Cambridge, \\ Wilberforce Road, Cambridge, CB3 0WA, U.K. \\ ${ }^{b}$ Centre for Advanced Mathematical Sciences and Physics Department, \\ American University of Beirut, Lebanon \\ E-mail: J.B.Gutowski@damtp.cam.ac.uk, ws00@aub.edu.1b
}

ERRATUM TO: JHEP12(2007)025

ABSTRACT: We present a systematic classification of half-supersymmetric solutions of gauged $N=2, D=5$ supergravity coupled to an arbitrary number of abelian vector multiplets for which at least one of the Killing spinors generate a time-like Killing vector.

The sentence after equation (5.89) should read: "Hence the 3-manifold with metric $\frac{1}{4}\left(\left(\sigma^{1}\right)^{2}+\left(\sigma^{2}\right)^{2}+\left(\sigma^{3}\right)^{2}\right)$ is either $\mathbb{S}^{3}$, the Nil-manifold or $S L(2, \mathbb{R})$ according as to whether $c \theta>0, \theta=0$ or $c \theta<0$ respectively."

The sentence after equation (5.102) should read: "Hence the 3-manifold with metric $\frac{1}{4}\left(\left(\sigma^{1}\right)^{2}+\left(\sigma^{2}\right)^{2}+\left(\sigma^{3}\right)^{2}\right)$ is either $\mathbb{S}^{3}$, the Nil-manifold or $S L(2, \mathbb{R})$ according as to whether $c \theta+9 \sqrt{2} \chi^{2}\left(X^{I} X^{J}-\frac{1}{2} Q^{I J}\right) V_{I} V_{J}<0, c \theta+9 \sqrt{2} \chi^{2}\left(X^{I} X^{J}-\frac{1}{2} Q^{I J}\right) V_{I} V_{J}=0$ or $c \theta+9 \sqrt{2} \chi^{2}\left(X^{I} X^{J}-\frac{1}{2} Q^{I J}\right) V_{I} V_{J}>0$ respectively." 